\title{
Triplet superconductivity induced by screened phonon interactions in ferromagnetic compounds
}

\author{
Hiroshi Shimahara and Mahito Kohmoto* \\ Department of Quantum Matter Science, ADSM, Hiroshima University, Higashi-Hiroshima 739-8530, Japan \\ * Institute of Solid State Physics, University of Tokyo, Kashiwa 277-8581, Japan
}

(Received January 2001)

\begin{abstract}
We propose that screened pairing interactions mediated by phonons could give rise to a spin triplet superconductivity in ferromagnetic compounds such as $\mathrm{UGe}_{2}$. It is pointed out that the pairing interactions include anisotropic components such as those of $p, d, f$-waves in addition to dominant $s$-wave component due to the momentum dependence. Since the ferromagnetic longrange order coexists, there is a large splitting of the Fermi surfaces of up and down spin electrons, which suppresses singlet pairing. Therefore, triplet pairing occurs at last due to the sub-dominant anisotropic interactions, even in the absence of magnetic contribution to the pairing interactions.
\end{abstract}

Recently, a coexistence of superconductivity and ferromagnetic long-range order was observed in $\mathrm{UGe}_{2}$ under pressure [1]. In the phase diagram on the pressure and temperature plane the superconductivity appears inside the area of the ferromagnetic phase. For this proximity of the superconductivity and the ferromagnetism, spin triplet superconductivity is a possible candidate in this compound.

In addition, singlet pairing is considered to be unfavalable in this compound. The ferromagnetism and superconductivity occur in the same electron band [1,2] or at least in very close electron bands from the crystal structure. Thus, there is a large Fermi surface splitting, which suppresses antiparallel spin pairing, in the electron band responsible to the superconductivity.

As a mechanism of triplet pairing, magnetically mediated superconductivity has been considered [1]. However, there are some behaviors which are not easily explained only by this mechanism. For example, the magnetic fluctuations and their contribution to the pairing interactions increase near the transition points. Such behavior is reproduced in the calculation of Fay and Appel [3]. In their calculation, it decreases in a narrow region of the width of $1 \%$ of the exchange interaction parameter near the second order transition point, but this decrease would not occur in $\mathrm{UGe}_{2}$, since the magnetic transition is of first order in $\mathrm{UGe}_{2}$. Therefore, the superconducting transition temperature seems to increase as the magnetic boundary is approached in this mechanism. However, in the observation, $T_{\mathrm{c}}$ decreases near the phase boundary $(\sim 1.6 \mathrm{GPa})$ within the width of $\sim 0.3 \mathrm{GPa}$ of the pressure, which is not very narrow.

Decreases of the superconducting transition temperatures as the magnetic phase approached were also observed in high- $T_{\mathrm{c}}$ superconductors. However, in those compounds, the magnetic phase is antiferromagnetism. Thus, we have a physical explanation of the decrease of the superconducting transition temperature based on the reduction of the density of states near the Fermi surface (pseudogap) due to the antiferromagnetic fluctuations [4]. On the other hand, the ferromagnetic fluc- tuations do not induce any pseudogap near the Fermi surface, since it is split to two surfaces.

On the other hand, if the decrease of the superconducting transition temperature near the magnetic phase boundary at the high pressure is correlated to the decrease of the ferromagnetic transition temperature, the absence of the superconductivity at pressures $p \lesssim 1 \mathrm{GPa}$ does not seem to be explained without any extra mechanisms. For example, pair breaking effect due to Lorentz force by internal magnetic field created by ferromagnetic moments might suppress the superconducting transition temperature at low pressure. It might be also possible that the degree of nesting of the Fermi surface [2] changes by pressure and gives rise to the pressure dependences of $T_{\mathrm{c}}$ and $T_{x}$ defined in [1]. Therefore, the magnetic mechanisms of the superconductivity is not conclusive at present.

In this paper, we propose a phonon mechanism of triplet superconductivity in $\mathrm{UGe}_{2}$ system. In the conventional mechanisms, the phonon mediated interactions have often been considered not to induce anisotropic superconductivity. However, we shall point out in this paper that the momentum dependence of the phonon mediated interactions could give rise to a triplet superconductivity in ferromagnetic superconductors. In a spin polarized state, the spin and charge degrees of freedom are locked together, and we concentrate ourselves on clarifying properties of the phonon mediated interaction.

It is easily verified that the pairing interactions contain both singlet and triplet components due to the momentum dependence. For example, when the screening length becomes longer for weak screening, the pairing interactions mediated by phonons have shaper peak near $q \sim 0$ in momentum space. Then, anisotropic components have large magnitudes. In the limit where the pairing interactions are proportional to the $\delta$-function, all components have the same magnitude. Foulkes and Gyorffy theoretically examined $p$-wave pairing in metals due to electron-phonon interaction, when the short range Coulomb interaction suppresses the $s$-wave pairing [5].

Anisotropic superconductivity induced by phonon in- 
teraction has been considered by Abrikosov as a mechanism of high- $T_{\mathrm{c}}$ superconductivity [6]. In this mechanism, it was shown that the gap function can vary in sign in the presence of on-site Coulomb repulsion. The momentum dependence of the pairing interactions was examined, where screening effect was taken into account. Bouvier and Bok calculated the superconducting gap and obtained anisotropic momentum dependence of the gap function [7] in the same model. Recently, Friedel and Kohmoto [8], and Chang, Friedel, and Kohmoto [9] have shown that $d$-wave superconductivity is induced by screened phonon interactions with an assist of a contribution from the antiferromagnetic fluctuations. In the studies so far, it was shown that a singlet pairing component is dominant in the screened phonon interactions.

Usually, since the largest component has even parity in the momentum space, it contributes to singlet pairing. Therefore, a sub-dominant triplet pairing interaction cannot induce superconducting transition. However, in ferromagnetic superconductors such as $\mathrm{UGe}_{2}$, the dominant singlet pairing interaction would not be able to overcome the Pauli pair breaking effect. Therefore, the sub-dominant interaction could give rise to the triplet superconductivity.

In the following, we illustrate that the phonon mediated pairing interactions contain both singlet and triplet pairing interactions by taking into account the screening effect. We calculate a coupling constant of sub-dominant triplet pairing interactions, which becomes dominant after singlet pairing is suppressed. It is shown that the screening effect gives rise to an additional lattice constant dependence of the pairing interactions through the screening length scaled by the inverse of the Fermi momentum.

We examine a model with a screened phonon interaction defined by

$$
V\left(\mathbf{k}, \mathbf{k}^{\prime}\right)=-g \frac{q_{s}{ }^{2}}{q^{2}+q_{s}^{2}} \cdot \frac{\omega(\mathbf{q})^{2}}{\omega(\mathbf{q})^{2}-\left(\xi_{\mathbf{k}}-\xi_{\mathbf{k}^{\prime}}\right)^{2}},
$$

where $\mathbf{q}=\mathbf{k}-\mathbf{k}^{\prime}, q=|\mathbf{q}|$, and $q_{s}{ }^{-1}$ is the screening length. This interaction has been examined by many authors [6] for the high- $T_{\mathrm{c}}$ superconductivity. As explained in the text books, near the Fermi surface where $|\xi| \lesssim \omega(\mathbf{q})$, the interaction is attractive due to overscreening. We put $\left(\xi_{\mathbf{k}}-\xi_{\mathbf{k}^{\prime}}\right)^{2} \sim 0$ for simplicity, since $k_{\mathrm{B}} T \ll \omega_{\mathrm{D}}$ where $\omega_{\mathrm{D}}$ denotes the Debye frequency. Hence, we have

$$
V\left(\mathbf{k}, \mathbf{k}^{\prime}\right)=-g \frac{q_{s}^{2}}{\left|\mathbf{k}-\mathbf{k}^{\prime}\right|^{2}+q_{s}^{2}} .
$$

Let us first consider the Thomas-Fermi screening for a while for a qualitative argument. We will improve our theory with a more detailed momentum dependence of the dielectric funcion in the random phase approximation (RPA) later. In the Thomas-Fermi approximation, we put

$$
q_{s}^{2}=4 \pi e^{2} \rho_{\mathrm{t}}(\mu)
$$

which is valid for long wave lengths such as $q \ll k_{\mathrm{F}}$. Here, $\rho_{\mathrm{t}}(\mu)$ is the total density of states of the conduction electrons per unit volume at the chemical potential $\mu$.

We assume a spherically symmetric Fermi surface for simplicity. Assuming $\omega_{\mathrm{D}} \ll \epsilon_{\mathrm{F}}$, we can put $|\mathbf{k}| \approx k_{\mathrm{F}}$. Thus, we have

$$
V\left(\mathbf{k}, \mathbf{k}^{\prime}\right)=-\frac{g(\alpha-1)}{\alpha-\cos \bar{\theta}}
$$

where $\bar{\theta}$ denotes the angle between $\mathbf{k}$ and $\mathbf{k}^{\prime}$, and $\alpha=$ $1+q_{s}^{2} / 2 k_{\mathrm{F}}^{2}$.

The pairing interaction eq. (4) is expanded as

$$
\begin{aligned}
V\left(\mathbf{k}, \mathbf{k}^{\prime}\right) & =\sum_{l=0}^{\infty} V_{l} P_{l}(\cos \bar{\theta}) \\
& =\sum_{l=0}^{\infty} \sum_{m=-l}^{l} g_{l} \gamma_{l m}(\theta, \varphi) \gamma_{l m}^{*}\left(\theta^{\prime}, \varphi^{\prime}\right),
\end{aligned}
$$

where $(\theta, \varphi)$ and $\left(\theta^{\prime}, \varphi^{\prime}\right)$ denote the directions of $\mathbf{k}$ and $\mathbf{k}^{\prime}$, respectively. Here, $g_{l}=V_{l} /(2 l+1)$ and $\gamma_{l m}(\theta, \varphi)=$ $Y_{l m}(\theta, \varphi)$ with the spherical harmonic functions $Y_{l m}$.

The $s$-wave coupling constant is calculated as

$$
\begin{aligned}
g_{0} & =V_{0}=\int V(\bar{\theta}) \frac{\mathrm{d} \bar{\Omega}}{4 \pi} \\
& =\frac{1}{2} g(\alpha-1) \log \left|\frac{\alpha-1}{\alpha+1}\right| .
\end{aligned}
$$

The coupling constant $g_{0}$ is larger than $g_{1}$, but it is not effective in practice, since singlet pairing is suppressed as we explained above. Thus, we calculate the next dominant $p$-wave component and obtain

$$
\begin{aligned}
g_{1} & =\frac{1}{3} V_{1}=\int V(\bar{\theta}) \cos \bar{\theta} \frac{\mathrm{d} \bar{\Omega}}{4 \pi} \\
& =g(\alpha-1)\left[1+\frac{\alpha}{2} \log \left|\frac{\alpha-1}{\alpha+1}\right|\right] .
\end{aligned}
$$

In order to improve our approximation, we examine the screening effect in an RPA. The pairing interaction is modifed as

$$
V\left(\mathbf{k}, \mathbf{k}^{\prime}\right)=-g \frac{q_{s}{ }^{2}}{q^{2}+q_{s}^{2} u\left(q / 2 k_{\mathrm{F}}\right)} \frac{\omega(\mathbf{q})^{2}}{\omega(\mathbf{q})^{2}-\left(\xi_{\mathbf{k}}-\xi_{\mathbf{k}^{\prime}}\right)^{2}}
$$

with

$$
u(x)=\frac{1}{2}\left[1+\frac{1-x^{2}}{2 x} \log \left|\frac{1+x}{1-x}\right|\right],
$$

according to the result of the dielectric function by Lindhard

$$
\kappa(q)=1+\frac{q_{s}^{2}}{q^{2}} u\left(\frac{q}{2 k_{\mathrm{F}}}\right) .
$$

The Thomas-Fermi approximation could be recovered in the long wave length limit. We apply the same simplification putting $\left(\xi_{\mathbf{k}}-\xi_{\mathbf{k}^{\prime}}\right)^{2} \sim 0$ in eq. (8). The coupling constant is obtained by numerical calculation. 
Figure 1 shows the dimensionless coupling constants defined by $\lambda_{p} \equiv\left|g_{1}\right| N(0)$, where $N(0)$ is the density of states per a spin and unit volume, in the Thomas-Fermi approximation and an RPA. It is found that they exhibit a peak around $q_{s}{ }^{2} / 2 k_{\mathrm{F}}^{2} \sim 1 / 2$, and the Thomas-Fermi approximation agrees well with the RPA for $q_{s}^{2} / 2 k_{\mathrm{F}}^{2} \lesssim$ $1 / 2$.

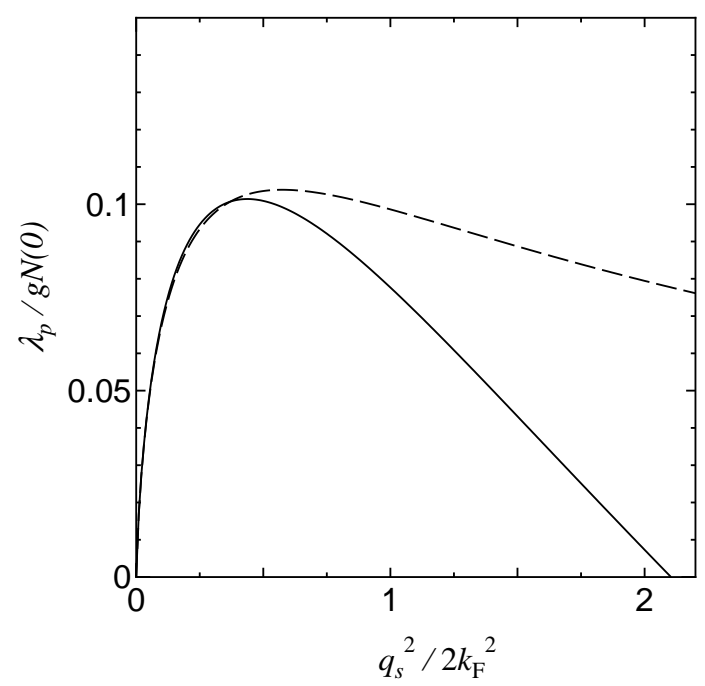

FIG. 1. Dimensionless coupling constant $\lambda_{p}$ of $p$-wave pairing in the unit of $g N(0)$ as a function of $q_{s}{ }^{2} / 2 k_{\mathrm{F}}^{2}$. Solid and dashed lines show results in the RPA and the Thomas-Fermi approximation, respectively.

The transition temperature of the $p$-wave superconductivity is given by

$$
T_{\mathrm{c} p}=1.13 \omega_{\mathrm{D}} \exp \left[-1 / \lambda_{p}\right]
$$

in the weak coupling limit. Short range Coulomb repulsion is not very effective in the case of $p$-wave pairing [5]. Figure 2 shows the transition temperature of the triplet superconductivity based on the formula (11). Because of the singular exponential form of eq. (11), the peak of $T_{\mathrm{c}}$ is sharper than that of $\lambda_{p}$.

The screening length $q_{s}^{-1}$ is considered as follows. The total density of states per unit volume is expressed as

$$
\rho_{\mathrm{t}}\left(\epsilon_{\mathrm{F}}\right)=2 \frac{m}{2 \pi^{2} \hbar^{2}} k_{\mathrm{F}} .
$$

Inserting eq. (12) into eq. (3), we have

$$
\alpha-1=\frac{q_{s}^{2}}{2 k_{\mathrm{F}}^{2}}=\frac{2}{\pi a_{\mathrm{H}}} \frac{1}{k_{\mathrm{F}}}
$$

where $a_{\mathrm{H}}$ denotes the Bohr radius $\left(a_{\mathrm{H}}=\hbar^{2} / e^{2} m \approx\right.$ $0.5293 \AA)$. Since $k_{\mathrm{F}} \propto 1 / a$ with lattice constant $a$, this expression means that a large lattice constant results in a strong screening effect, (i.e., a short screening length $\left.q_{s}{ }^{-1}\right)$.

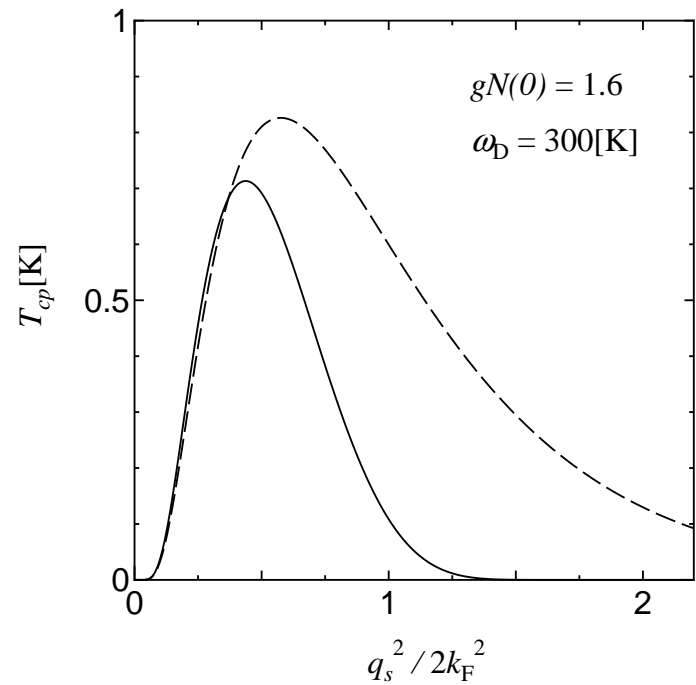

FIG. 2. Superconducting transition temperature $T_{\mathrm{c} p}$ of $p$-wave pairing as a function of $q_{s}^{2} / 2 k_{\mathrm{F}}^{2}$ calculated by eq. (11). $\omega_{\mathrm{D}}=300[\mathrm{~K}]$ and $g N(0)=1.6$ are assumed as an example. Solid and dashed lines show results in the RPA and the Thomas-Fermi approximation, respectively.

In our spherically symmetric system, the Fermi momentum $k_{\mathrm{F}}$ is expressed as $k_{\mathrm{F}}=\left(3 \pi^{2}\right)^{1 / 3} n^{1 / 3} / a$, where $n$ denotes conduction electron (or hole) number per a site. Thus, eq. (13) is written as

$$
\frac{q_{s}^{2}}{2 k_{\mathrm{F}}^{2}}=\frac{2}{\pi\left(3 \pi^{2}\right)^{1 / 3} n^{1 / 3}} \frac{a}{a_{\mathrm{H}}} \equiv \frac{a}{a_{0}} .
$$

Here, we have defined a characteristic length $a_{0}$ by

$$
a_{0}=\frac{1}{2} \pi\left(3 \pi^{2}\right)^{1 / 3} n^{1 / 3} a_{\mathrm{H}},
$$

which scales the lattice constant. If we put $n \sim 1$ as the order of magnitude, we obtain $a_{0}=2.57 \AA$. When $a \sim 4 \AA$ [10, 11], one has $q_{s}^{2} / 2 k_{\mathrm{F}}^{2}=a / a_{0} \sim 1.6$ as the order of magnitude.

Now, we discuss the pressure dependence of the transition temperature of the triplet supercoductivity. The pressure dependence of the transition temperature is not easily figured out theoretically in the phonon mechanisms even in $s$-wave superconductors. The Debye frequecy $\omega_{\mathrm{D}}$ increases with pressure. Then, the prefactor of the weak coupling expression of $T_{\mathrm{c}}$ increases. On the other hand, the electron-phonon coupling constant and the electron density of states would decrease. These effects would contribute to the the pressure dependence in conventional superconductors. In $\mathrm{UGe}_{2}$, however, the superconducting transition temperature increases more sensitively to the pressure near $1 \mathrm{GPa}$ [1] than those in conventional superconductors. 


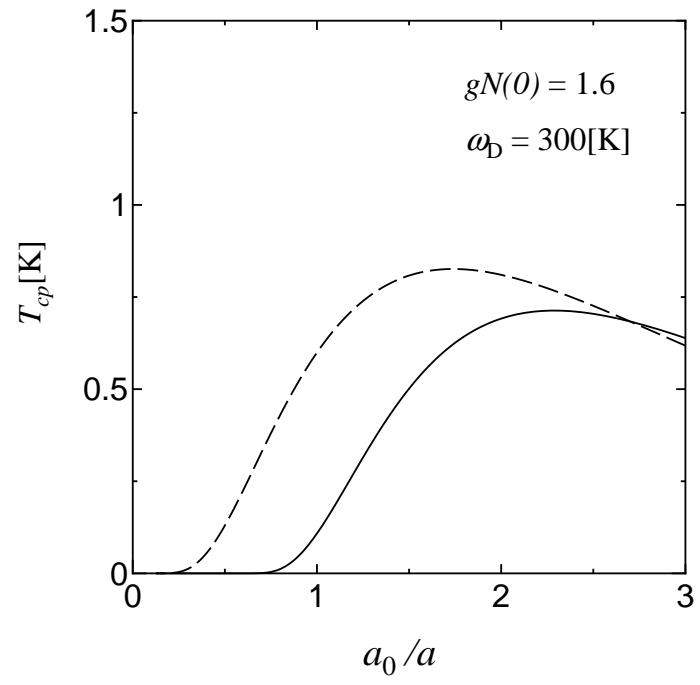

FIG. 3. Superconducting transition temperature $T_{\mathrm{c} p}$ of $p$-wave pairing as a function of $a_{0} / a$ calculated by eq. (11). $\omega_{\mathrm{D}}=300[\mathrm{~K}]$ and $g N(0)=1.6$ are assumed as an example. Solid and dashed lines show results in the RPA and the Thomas-Fermi approximation, respectively.

In the present mechanism, we have an additional contribution to the pressure dependence through the screening effect. At high pressures, the lattice constant $a$ is shorten. Then, from eq. (14), the value of $q_{s}^{2} / 2 k_{\mathrm{F}}^{2}$ is reduced by the pressure. Figure 3 shows dependence of the superconducting transition temperature on the inverse of the lattice constant. A large value of $1 / a$ corresponds to a high pressure. Since the pressure dependences of $\omega_{\mathrm{D}}$, $g, N(0)$ are not considered, this figure only shows the additional contribution though the screening effect. It is found that the superconductivity does not occur for $a \gtrsim a_{0}$ and that the pressure would enhance the transition temperature of the triplet superconductivity near $a \sim a_{0}$. This result is consistent with the behavior of the transition temperature in the experimental phase diagram near $1 \mathrm{GPa}$ qualitatively, although it is more sensitive to the pressure in the experiment.

In addition, inside the region of the ferromagnetic phase, Lorentz force created by internal magnetic field should suppress pair formation. The superconducting transition temperature should be reduced from the value that we estimated in the above. Thus, the superconductivity must be absent in a wider region than those in Fig. 2 and Fig. 3 .

On the other hand, decreases of the superconducting and the ferromagnetic transition temperatures are observed at high pressures $(\sim 1.6 \mathrm{GPa})[1]$. For such high pressures, crystal structure might become unstable. Single phases might not be able to exist in this region. The decreases of the transition temperatures might be due to the instability of the crystal. At very high pressures, an unharmonicity may play some role in the pressure dependence of the superconducting transition temperature.

In addition, near the phase boundary between the fer- romagnetic and paramagnetic states, the ferromagnetic fluctuations should be strong. The superconductivity might be suppressed near the phase boundary by some renormalization effect due to the strong magnetic fluctuations, since the pairing interactions would not increase significantly there in phonon mechanisms. In a spin polarized state the spin and charge degrees of freedom are locked together. The pressure dependence of $T_{\mathrm{c}}$ and $T_{x}$ might be explained by taking into account the change in the degree of nesting of the quasi-two-dimensional parts of the Fermi surface [2].

In conclusion, we proposed a phonon mechanism of triplet superconductivity in ferromagnetic systems. The momentum dependence of the pairing interactions mediated by phonons give rise to both singlet and triplet components. The singlet interaction is stronger than the triplet one, but the Fermi surface splitting suppresses the singlet superconductivity in the ferromagnetic state. For an appropriate region of the screening length, the triplet superconductivity occurs. The superconducting transition temperature depends on the lattice constant through the screening length scaled by inverse of the Fermi momentum. Such dependence might contribute to the pressure dependence of the superconducting transition temperature in $\mathrm{UGe}_{2}$.

This work was partially (H.S.) supported by a grant for Core Research for Evolutionary Science and Technology (CREST) from Japan Science and Technology Corporation (JST).

[1] S. S. Saxena, et al., Nature 406 (2000) 587.

[2] A. B. Shick and W. E. Pickett, Phys. Rev. Lett. 86 (2001) 300.

[3] D. Fay and J. Appel, Phys. Rev. B 22 (1980) 3173.

[4] H. Shimahara, Y. Hasegawa, and M. Kohmoto, J. Phys. Soc. Jpn. 69 (2000) 1598, and references therein.

[5] I. F. Foulkes and B. L. Gyorffy, Phys. Rev. B 15 (1977) 1395.

[6] A. A. Abrikosov, Physica C 222 (1994) 191; ibid 244 (1995) 243.

[7] J. Bouvier and J. Bok, Physica C 249 (1995) 117.

[8] J. Friedel and M. Kohmoto, Int. J. Mod. Phys. B 15 (2001) 511.

[9] I. Chang, J. Friedel, and M. Kohmoto, Europhys. Lett. 50 (2000) 782.

[10] E. S. Makarov and V. N. Bykov: Kristallografiya 4 (1959) 183.

[11] K. Oikawa, T. Kamiyama, H. Asano, Y. Ōnuki, and M. Kohgi, J. Phys. Soc. Jpn. 65 (1996) 3229. 